\title{
铁磁性材料早期疲劳损伤磁混频检测方法*
}

\author{
焦敬品 ${ }^{1}$ 常 予 $^{1}$ 李光海 $^{2}$ 吴 斌 ${ }^{1}$ 何存富 ${ }^{1}$ \\ (1. 北京工业大学机械工程与应用电子技术学院 北京 100124; \\ 2. 中国特种设备检测研究院 北京 100013)
}

\begin{abstract}
摘要: 在金属材料常见的早期力学性能退化损伤中, 疲劳对结构的潜在危害性最大。基于铁磁性材料的磁滞非线性特性, 在 高低频叠加励磁条件下, 研究铁磁材料中磁混频效应的产生机理及表现形式。分析混频分量和局部磁滞回线与材料早期损伤 的关系，提出磁混频非线性因子与局部磁滞损耗幂律系数两种特征参量用于铁磁性材料磁混频效应的表征。在此基础上，将 磁混频检测技术用于铁磁性材料疲劳损伤检测。结果表明, 提出的两种磁混频特征参量可用于铁磁性材料早期瘦劳损伤的表 征。为铁磁性材料早期疲劳损伤检测提供了可行的技术方案。
\end{abstract}

关键词: 铁磁性材料; 疲劳损伤; 磁混频; 磁滞非线性; 幂律系数

中图分类号: TM154

\section{Magnetic Mixing Detection Method for Early Fatigue Damage of Ferromagnetic Materials}

\author{
JIAO Jingpin ${ }^{1}$ CHANG Yu ${ }^{1} \quad$ LI Guanghai ${ }^{2} \quad$ WU Bin $^{1} \quad \mathrm{HE} \mathrm{Cunfu}^{1}$ \\ (1. College of Mechanical Engineering and Application Electronics Technology, \\ Beijing University of Technology, Beijing 100124; \\ 2. China Special Equipment Inspection and Research Institute, Beijing 100013)
}

\begin{abstract}
Among the common early mechanical property degradation damage of metal materials, fatigue has the greatest potential harm to the structure. Based on the hysteresis nonlinearity of ferromagnetic materials, the mechanism and manifestation of magnetic frequency mixing effect in ferromagnetic materials under high and low frequency excitation are analyzed. The relationship between the mixing component, the local hysteresis loops and the early damage of magnetic ferromagnetic materials is analyzed. The magnetic mixing nonlinear factor and the power law coefficient of local hysteresis loss are proposed to characterize the magnetic frequency mixing effect of ferromagnetic materials. Then, magnetic frequency mixing detection technology is used to detect fatigue damage of ferromagnetic materials. Experimental results show that the early fatigue damage of ferromagnetic materials can be characterized by two kinds of magnetic mixing characteristic parameters. This research work provides a feasible technical scheme for early fatigue damage detection of ferromagnetic materials.
\end{abstract}

Key words: ferromagnetic material; fatigue; magnetic mixing frequency; magnetic nonlinear; power law coefficient

\section{0 前言}

作为一种常见的承载构件, 铁磁性材料广泛应 用于航空航天、电力、铁路和压力容器等行业。这 些构件在使用过程中, 由于循环载荷、内部工作介 质和外部环境因素的作用，易在结构的不均匀处、 疏松区域、空洞及位错缺陷的集中区产生疲劳损伤, 导致疲劳断裂 ${ }^{[1]}$ 。因此，对铁磁性构件的疲劳损伤

* 国家重点研发计划(2017YFF0209703)和国家自然科学基金(11527801, 11572010 )资助项目。20190408 收到初稿, 20191023 收到修改稿
进行及时、有效的检测, 对于预防承载构件的断裂 和防止重大安全事故的发生具有重要意义。

现阶段, 检测疲劳损伤的常用手段主要包括红 外热成像法 ${ }^{[2]}$ 、脉冲涡流热成像 ${ }^{[3]}$ 和声发射法 ${ }^{[4]}$ 。但 这些方法对裂纹萌生前的早期疲劳损伤敏感度较 低。基于循环载荷作用下材料具有的迟滞非线性效 应，国内外学者将超声谐波法技术应用于结构早期 疲劳损伤检测 ${ }^{[5]}$ 。但该技术受检测系统非线性效应 的影响大, 难以区分出检测结果中非线性效应的来 源 $^{[6]}$ 。基于铁磁性材料的磁致伸缩特性, 国内外学者 研究利用金属磁记忆法对结构疲劳损伤进行检测 ${ }^{[7]}$, 
但该方法检测能力有限, 难以实现结构早期疲劳损 伤的检测。

通常, 当铁磁材料处于外加磁场作用下时, 磁 畴及磁畴壁的运动会不断克服材料内部的能量壁 垒, 使材料具有磁滞特性 ${ }^{[8]}$ 。磁滞特性可表现为多 种弱磁非线性效应, 如磁畴旋转非线性效应、磁畴 壁移动非线性效应和磁滞损耗非线性效应等 ${ }^{[9]}$ 。当铁 磁性构件处于疲劳早期时, 结构内部微损伤会影响 能量壁垒, 导致其弱磁非线性效应的变化 ${ }^{[10-11]}$ 。针对 铁磁性构件早期损伤检测问题, 国内外学者研究了 铁磁性构件损伤与上述非线性磁效应的关系, 发展 了多种微磁无损检测方法。例如, SAMIMI 等 ${ }^{[12]}$ 将 磁畴壁移动非线性效应产生的巴克豪森噪声用于钢 结构中残余应力表征。MATSUMOTO 等 ${ }^{[13]}$ 研究了 可逆磁化条件下磁畴的旋转非线性效应。发现可 逆磁化下的增量磁导率对材料相含量的变化十分 敏感, 并将该参数用于铸铁硬度变化的表征。 CHEN 等 ${ }^{[14-15]}$ 分析了增量磁导率随材料力学性能 的变化规律, 通过试验验证了增量磁导率对碳钢塑 性变形表征的有效性。ZIRKA 等 ${ }^{[16]}$ 分析了磁化条 件对磁滞损耗非线性效应的影响, 利用脉宽调制 信号励磁, 建立了包含局部磁滞回线的磁滞模型, 从理论上证明了局部磁滞损耗的非线性特性。

KOBAYASHI 等 ${ }^{[17-18]}$ 研究了磁滞损耗与材料力学 性能的关系, 提出了利用局部磁滞损耗幂律系数表 征材料力学性能变化的方法, 并将其用于结构早期 蠕变及辐照退化的表征。

除上述常规的微磁检测技术外, 磁混频检测是 近年来发展起来的一种基于铁磁性材料混频非线性 效应的新型微磁无损检测技术 ${ }^{[19]}$ 。在磁混频检测 中, 铁磁材料通常处于高低频叠加的交变磁场中。 其中, 低频磁场强度较大, 可将构件磁化至近饱和 状态, 高频磁场强度较小, 仅在构件近表面形成弱 扰动磁场 ${ }^{[20]}$ 。在两磁场的共同作用下, 铁磁材料中 磁畴的旋转与畴壁的移动会产生磁混频效应 ${ }^{[21-22]}$, 混频效应也是铁磁材料微观磁滞损耗非线性特性的

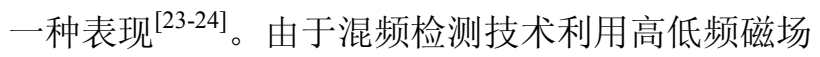
叠加产生的高频分量进行结构微磁特性的表征, 使 得磁混频检测技术具有检测灵敏度和信噪比高的特 点。基于磁混频效应, KRAUSE 等 ${ }^{25]}$ 进行了磁混频检 测技术研究, 开发了专用的金属离子磁混频检测装 置, 实现了水中铁粒子浓度的检测。TELIBAN 等 ${ }^{[26]}$ 研究了多阶混频分量幅值随涂层厚度的变化规律, 利用高阶混频分量实现了纳米级涂层厚度表征。TU 等 ${ }^{[27]}$ 研究了磁混频效应随纳米粒子布朗运动的变 化规律, 利用磁混频技术实现了流体中磁纳米粒子
粒径的表征。综上所述, 目前磁混频检测技术的研 究还处于起步阶段, 其研究主要集中于生物医学领 域中微纳米磁粒子及磁薄膜的检测, 其在无损检测 领域的应用还鲜有报道。

鉴于磁混频检测技术对结构微磁特性的高敏感 性, 本文将磁混频技术应用于铁磁性材料早期疲劳 损伤检测。基于磁滞模型, 进行了磁混频检测原理 分析, 研究了混频激励下铁磁性材料混频效应的表 现形式, 提出了可用于铁磁性材料磁混频效应表征 的两种特征参数。在此基础上, 进行了铁磁材料早 期疲劳损伤磁混频检测试验, 验证了两种表征参数 对结构疲劳损伤表征的有效性。

\section{1 磁混频检测方法}

高低频叠加励磁是磁混频检测的基础。基于磁 化场理论, 本部分对高低频叠加励磁条件下的磁混 频效应进行研究, 提出可用于混频效应表征的特征 参数。

\section{1 磁混频检测原理}

在外加磁场作用下, 铁磁材料的磁化强度 $M$ 可 表示为

$$
M\left(\mu_{0} H(t)\right)=M_{\mathrm{s}} \cdot \mathcal{L}\left(\frac{m_{0} \mu_{0} H(t)}{k_{B} T}\right)
$$

式中, $M_{\mathrm{s}}$ 为饱和磁化强度, $m_{0}$ 为磁矩, $\mu_{0}$ 为真空磁 导率, $H$ 为外加磁场, $k_{B}$ 为玻尔兹曼常数, $T$ 为开 尔文温度, $\mathscr{L}($ ) 为郎之万函数, 其一般式可表示为

$$
\mathscr{L}(x)=\operatorname{coth}(x)-\frac{1}{x}
$$

磁混频效应是高、低频励磁场共同作用于铁磁 性材料时产生的一种电磁非线性效应。当作用在铁 磁材料上的外加磁场 $H$ 为包含两不同激励频率 $\left(f_{1}\right.$ 和 $f_{2}$ ) 的励磁场时, 由于材料的磁滞特性, 两磁场将 产生耦合作用。通常, 混频磁化场 $H(t)$ 可表示为

$$
H(t)=A_{1} \sin \left(2 \pi f_{1} t+\phi_{1}\right)+A_{2} \sin \left(2 \pi f_{2} t+\phi_{2}\right)
$$

式中, $\phi_{1}$ 和 $\phi_{2}$ 分别为高低频分量的频率, $A_{1}$ 和 $A_{2}$ 分别为高低频分量幅值, $t$ 为时间。假设上式中 $f_{1}>f_{2}$, 根据式(2)中的郎之万方程, 式(1)中磁化强度的泰勒 级数展开式可表示为

$$
\begin{gathered}
M(t) \approx \frac{M_{\mathrm{s}}}{3} \frac{m_{0} \mu_{0} H(t)}{k_{\mathrm{B}} T}-\frac{M_{\mathrm{s}}}{45}\left(\frac{m_{0} \mu_{0}}{k_{\mathrm{B}} T}\right)^{3} H^{3}(t)+\cdots= \\
\frac{M_{\mathrm{s}}}{3} \frac{m_{0} \mu_{0}}{k_{\mathrm{B}} T}\left[A_{1} \sin \left(2 \pi f_{1} t+\phi_{1}\right)+\right. \\
\left.A_{2} \sin \left(2 \pi f_{2} t+\phi_{2}\right)\right]+\cdots+
\end{gathered}
$$




$$
\begin{gathered}
\frac{M_{\mathrm{s}}}{180}\left(\frac{m_{0} \mu_{0}}{k_{\mathrm{B}} T}\right)^{3}\left[A_{1}^{3} \sin \left(2 \pi\left(3 f_{1}\right) t+3 \phi_{1}\right)+\right. \\
\left.A_{2}^{3} \sin \left(2 \pi\left(3 f_{2}\right) t+3 \phi_{2}\right)\right]+\cdots+ \\
\frac{M_{\mathrm{s}}}{60}\left(\frac{m_{0} \mu_{0}}{k_{\mathrm{B}} T}\right)^{3} A_{1} A_{2}^{2} \sin \left(2 \pi\left(f_{1} \pm 2 f_{2}\right) t+\right. \\
\left.\phi_{1} \pm 2 \phi_{2}\right)+\cdots
\end{gathered}
$$

对式(4)进行傅里叶变换, 得到

$$
\begin{gathered}
M(f)=\mathrm{j} \frac{\alpha M_{\mathrm{s}} A_{1}}{6} \delta\left(f_{1}-f\right) \exp \left(\mathrm{j} \phi_{1}\right)+ \\
\mathrm{j} \frac{\alpha M_{\mathrm{s}} A_{2}}{6} \delta\left(f_{2}-f\right) \exp \left(\mathrm{j} \phi_{2}\right)+\cdots+ \\
\mathrm{j} \frac{\alpha^{3} M_{\mathrm{s}} A_{1}^{3}}{360} \delta\left(3 f_{1}-f\right) \exp \left(\mathrm{j} \phi_{1}\right)+ \\
\mathrm{j} \frac{\alpha^{3} M_{\mathrm{s}} A_{2}^{3}}{360} \delta\left(3 f_{2}-f\right) \exp \left(\mathrm{j} \phi_{2}\right)+\cdots+ \\
\mathrm{j} \frac{\alpha^{3} M_{\mathrm{s}} A_{1} A_{2}^{2}}{120} \delta\left(f_{1} \pm 2 f_{2}-f\right) \exp \left[\mathrm{j}\left(\phi_{1} \pm 2 \phi_{2}\right)\right]+\cdots
\end{gathered}
$$

式中, $\alpha=m_{0} \mu_{0} / k_{B} T$ 。由式(4)和式(5)可知, 响应信号 中不仅出现了 $f_{1}$ 和 $f_{2}$ 的线性响应分量, 还出现了谐 波分量(如 $\left.3 f_{1} 、 3 f_{2}\right)$ 和混频分量 $\left(f_{1} \pm 2 f_{2}\right)$ 等非线性响应 分量。值得注意的是, 由于对称相消法则, 响应中 只出现了多阶奇次谐波项和偶次混频项。

\section{2 磁混频效应表征参数}

磁混频检测中, 利用设计的磁混频传感器同时 拾取待检测试件中的磁感应信号和切向磁场信号。 基于这两类信号, 本节进行了磁混频效应表征参数 提取, 图 1 给出了磁混频检测信号分析方法的流程 图。可以看出, 信号分析主要涉及两方面。一方面, 对磁感应强度信号进行频谱分析, 从其频谱中提取 出磁混频非线性因子; 另一方面, 以切向磁场信号 作为自变量, 以磁感应强度作为因变量, 得到混频 励磁下的磁滞回线, 从中提取出局部磁滞损耗幂律 系数。下面给出两种特征参数的具体表达式。

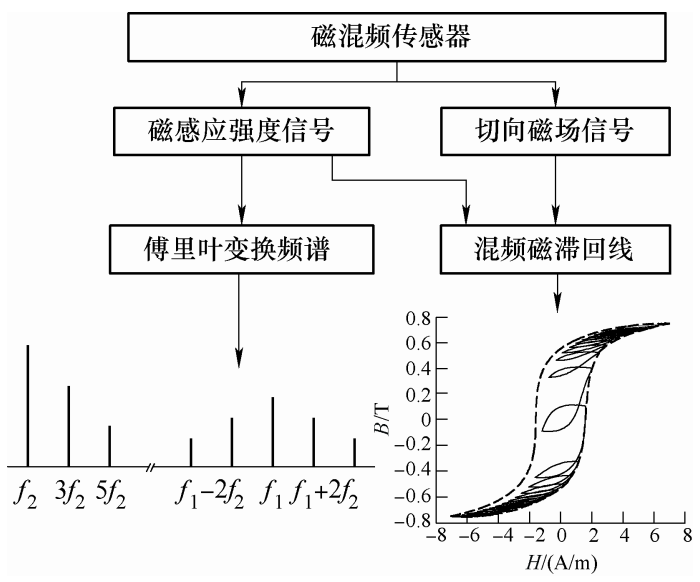

图 1 磁混频检测信号分析方法示意图

\subsection{1 磁混频非线性因子}

根据式(5)给出的混频信号的频域表达式可知, 混频效应主要体现在混频分量(如 $f_{1} \pm 2 f_{2}$ ) 的强弱, 而 混频分量与高频分量所处频带接近(图 2), 因此响应信 号中高频分量的幅值会影响混频效应的对比度。借鉴 非线性超声检测领域中振动声调制检测中对非线性 效应的表征方法, 定义磁混频非线性因子为

$$
M=\frac{A_{f_{1}-2 f_{2}}+A_{f_{1}+2 f_{2}}}{A_{f_{1}}}
$$

式中, $A_{f 1-2 f 2} 、 A_{f 1+2 f 2}$ 和 $A_{f 1}$ 分别为差频分量、和频 分量及高频基频分量的幅值。磁混频非线性因子可 直接给出各混频分量与高频分量的关系，也可直观 反映混频分量幅值强度的变化情况。

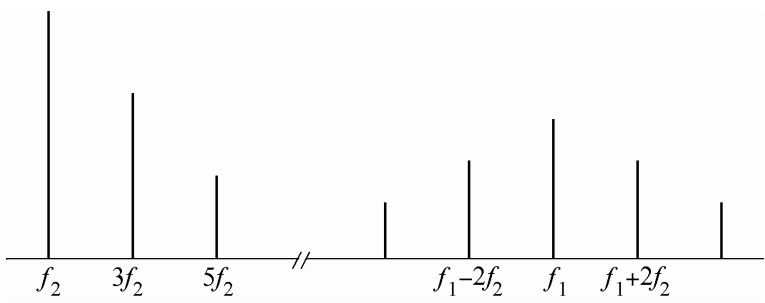

图 2 混频检测信号频谱示意图

\subsection{2 局部磁滞损耗幂律系数}

在混频励磁条件下，铁磁材料的混频磁滞回线 是由高、低频磁化场作用下的主磁滞回线及局部磁 滞回线组成的，如图 3 所示。其中，局部磁滞回线 是磁混频效应的一种表现，它所包围的面积可表征 材料的局部磁滞损耗。

根据斯坦梅茨磁滞损耗理论, 磁滞损耗与磁通 量密度间的幂律关系同样适用于局部磁滞回线, 可 表示为

$$
W_{\mathrm{F}}^{*}=W_{\mathrm{F}}^{0}\left(\frac{B_{\mathrm{a}}^{*}}{B_{\mathrm{S}}}\right)^{n_{\mathrm{F}}}
$$

式中, $W_{\mathrm{F}}{ }^{*}$ 为局部磁滞损耗, 可由图 $3 \mathrm{c}$ 中局部磁滞 回线的面积表示。 $B_{\mathrm{a}}{ }^{*}$ 为最大磁通量密度, 可由图 $3 \mathrm{c}$

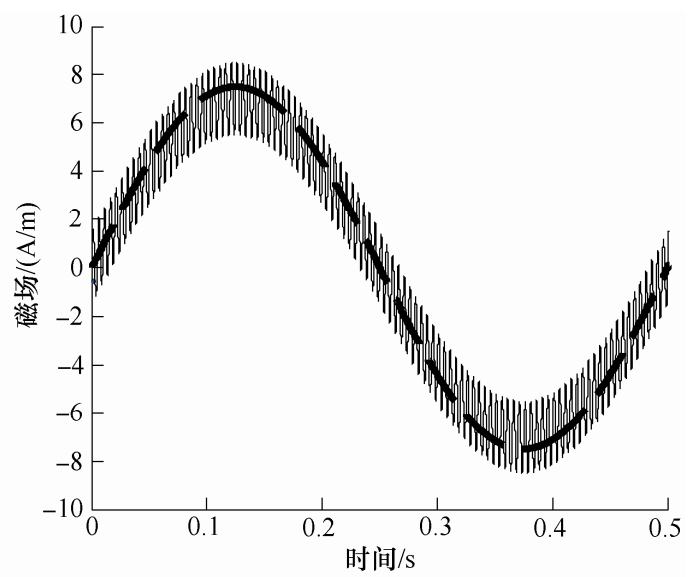

(a) 混频励磁信号 


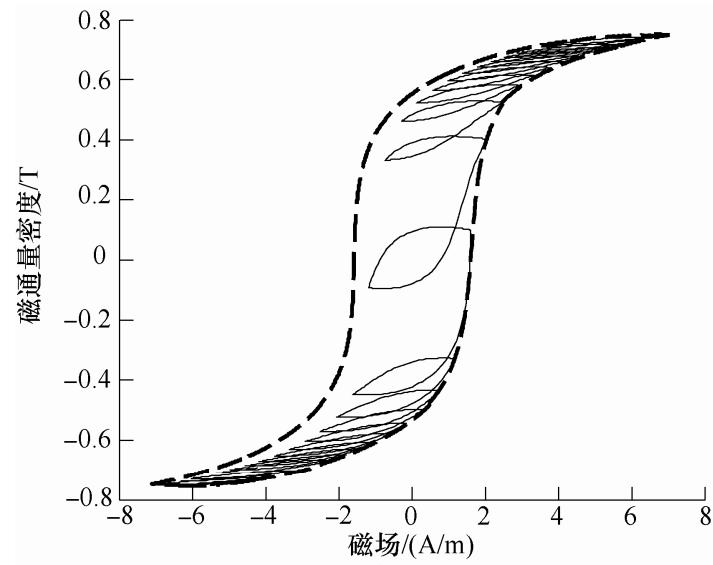

(b) 混频磁滞回线

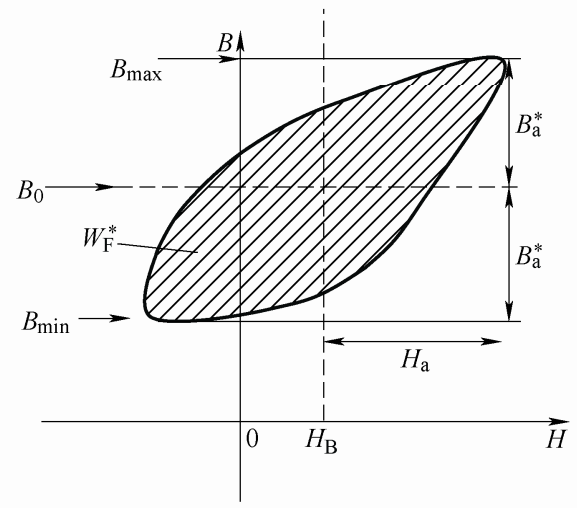

(c) 局部磁滞损耗幂律关系

图 3 铁磁材料混频磁化的磁滞回线示意图

中局部磁滞回线的磁通密度峰值计算, 即 $B_{\mathrm{a}}{ }^{*}=$ $\left|B_{\max }-B_{\min }\right| 2$ 。 $B_{\mathrm{s}}$ 为主磁滞回线的饱和磁通量密度。 $n_{\mathrm{F}}$ 为幂指数常数, 铁基合金材料的幂指数通常为 1.5 或 1.6。根据局部磁滞回线, 获得式(7)中的局部磁 滞损耗幂律系数 $W_{\mathrm{F}}^{0}$ 。该系数反映了磁混频效应中 局部磁滞损耗随动态磁化场的变化情况。

\section{2 磁混频疲劳检测试验}

为了说明磁混频检测技术对早期疲劳损伤检测 的有效性, 利用图 4 所示的磁混频检测系统对一批 具有不同程度疲劳的铁磁试件进行了检测试验。该 检测系统主要由激励采集板卡、功率放大器、磁混

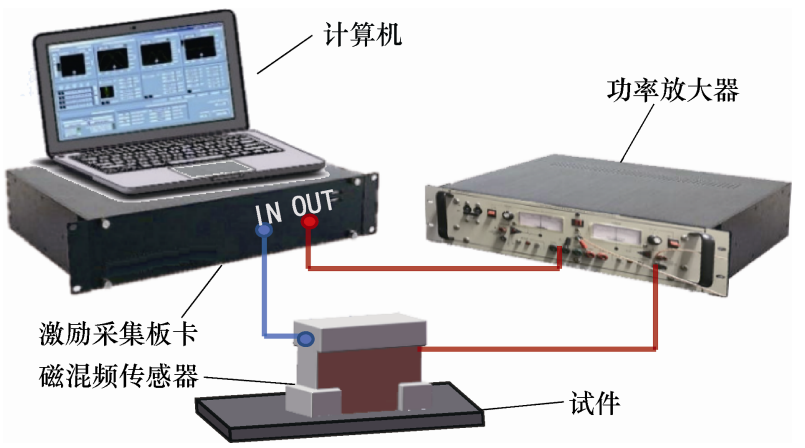

图 4 磁混频检测试验系统
频传感器、被测试件和电脑等组成。其中, 磁混频 传感器主要包括 U 型磁芯、激励线圈、检测线圈和 霍尔元件。根据磁混频激励参数的优选结果, 磁混 频疲劳损伤检测试验所用激励参数为高频激励频率 $200 \mathrm{~Hz}$ ，低频激励频率 $1 \mathrm{~Hz}$, 高频激励幅值 $1 \mathrm{~V}$, 低频激励幅值 $5 \mathrm{~V}$ 。

待检测疲劳试件材料为 45 钢, 其热处理过程包 括在 $830 \sim 850{ }^{\circ} \mathrm{C}$ 淬火和 $550 \sim 600{ }^{\circ} \mathrm{C}$ 回火后, 进行 油冷。将热处理后钢板切割成标准拉伸试件, 其几 何尺寸和性能参数分别如图 5 和表 1 所示。

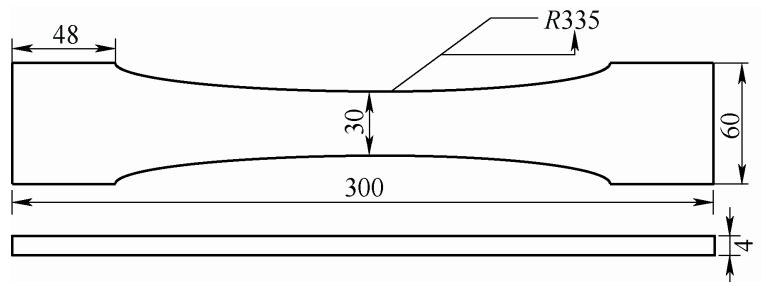

图 5 疲劳试件尺寸 $(\mathrm{mm})$

表 145 号钢试件材料参数

\begin{tabular}{cccccccc}
\hline & \multicolumn{3}{c}{ 化学成分 $(\%)$} & & $\begin{array}{c}\text { 屈服强度/ } \\
\mathrm{MPa}\end{array}$ & $\begin{array}{c}\text { 抗拉强度/ } \\
\mathrm{MPa}\end{array}$ & $\begin{array}{c}\text { 弹性模量/ } \\
\mathrm{GPa}\end{array}$ \\
\hline $\mathrm{C}$ & $\mathrm{Mn}$ & $\mathrm{S}$ & $\mathrm{Si}$ & $\mathrm{Ma}$ & \\
\hline 0.47 & 0.59 & 0.0058 & 0.20 & 404 & 557 & 175 \\
\hline
\end{tabular}

采用 QBG-200 型高频疲劳试验机对这批试件 进行高周疲劳试验。根据待检测试件的力学性能, 疲劳试验参数设定为静载荷 $25.83 \mathrm{kN}$, 动载荷 25.83 $\mathrm{kN}$, 应力比为 0 。循环加载波形为正弦波, 频率为 $125 \mathrm{~Hz}$ 。通过多次重复测试, 测得本研究所用 45 钢 在当前高频疲劳加载条件下的疲劳寿命约为 40 万 次。在上述疲劳加载条件不变的条件下对不同试件 进行不同周期数的疲劳加载, 以获得不同疲劳程度 的试件, 如图 6 所示。表 2 给出了各试件的疲劳周 期数。

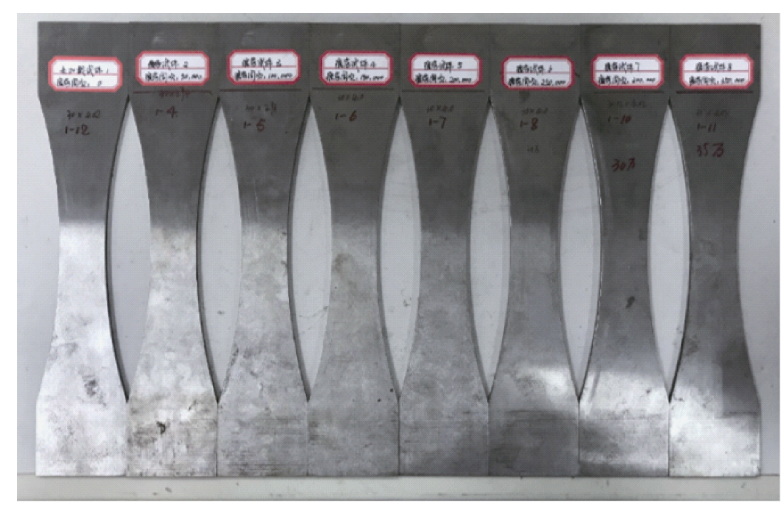

图 6 不同加载周期数的早期疲劳试件

表 2 试件的疲劳周期

\begin{tabular}{ccccccccc}
\hline 序号 & 1 & 2 & 3 & 4 & 5 & 6 & 7 & 8 \\
\hline 疲劳周期 $/ 10^{3}$ 次 & 0 & 50 & 100 & 150 & 200 & 250 & 300 & 350 \\
\hline
\end{tabular}


将各试件在疲劳加载后静置 $48 \mathrm{~h}$ 以上后, 利用 图 4 中的磁混频检测系统对其进行离线检测试验。 对每个试件进行五次重复试验, 每次在试件三个不 同的位置进行检测试验。图 7 给出了磁混频传感器 采集到的两类信号的典型波形。

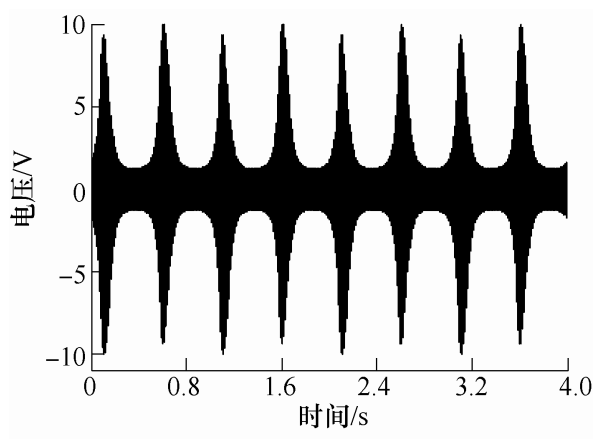

(a) 试件内磁通量密度

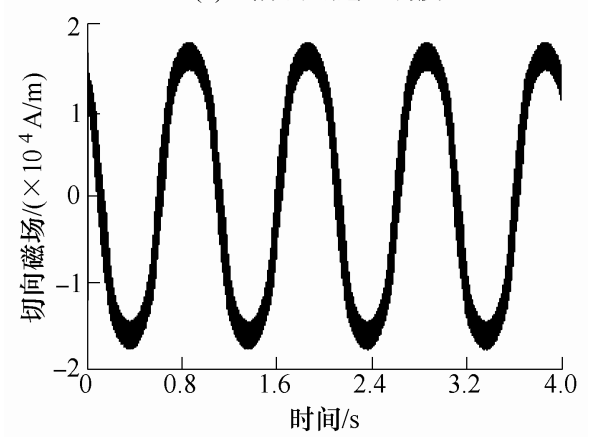

(b) 试件表面切向磁场

图 7 离线情况下, 磁混频传感器采集到的典型检测信号

图 7a 为线圈采集到的试件内磁通密度的电压 信号, 图 7b 为霍尔元件采集到的切向磁场信号。两 信号的采集过程为同步采集, 采样率为 $2.5 \times 10^{4}$, 采样点数均为 $10^{5}$, 采集周期数为 4 。由两信号可知, 磁通密度的峰值位置对应切向磁场的过零点位置。 混频传感器采集到的这两类信号将用于混频特征参 数的计算。

为进一步反映混频检测技术对疲劳损伤检测的 有效性, 在疲劳试件制备过程中也对三个试件进行 了在线混频检测试验。三个试件出现㽻劳裂纹时的 周期数分别是 $396.7 \times 10^{3}$ 次、 $300.8 \times 10^{3}$ 次和 434.1 $\times 10^{3}$ 次。当试验系统与混频检测参数不变时, 高周 疲劳试验机每加载 5 万次暂停, 对疲劳试件进行磁 混频检测。磁混频传感器垂直于试件表面, 分别在 三个位置采集混频信号, 提离距离为 $0.2 \mathrm{~mm}$, 如图 $8 \mathrm{a}$ 所示, 检测过程中试件一直处于加持状态。当疲 劳周期达到 43.6 万次时, 试件中心出现㽻劳裂纹, 如图 $8 \mathrm{~b}$ 所示, 此时疲劳试验终止。磁混频传感器采 集到的试件内磁通量密度和试件表面切向磁场信号 如图 9 所示。

可以看出, 在线情况下磁混频传感器检测信号 与离线检测信号基本一致, 但两类信号的信噪比明

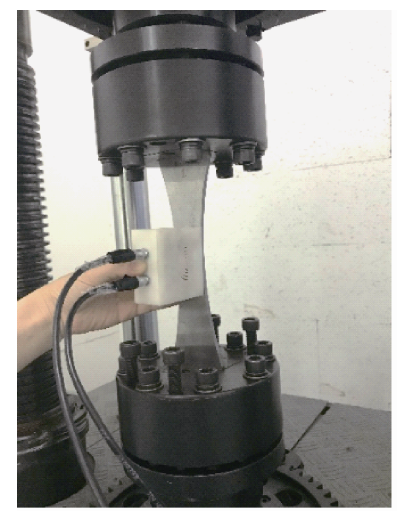

(a) 传感器采集信号位置

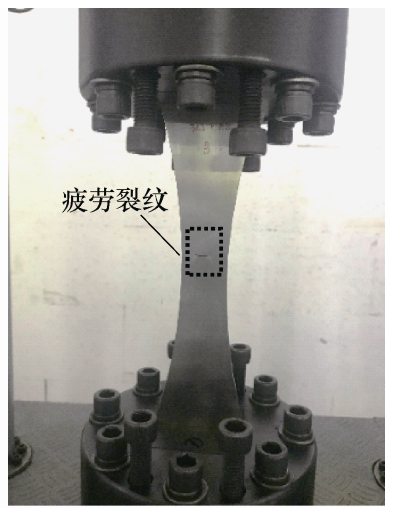

(b) 疲劳裂纹萌生位置
图 8 磁混频疲劳在线检测试验测试及裂纹位置

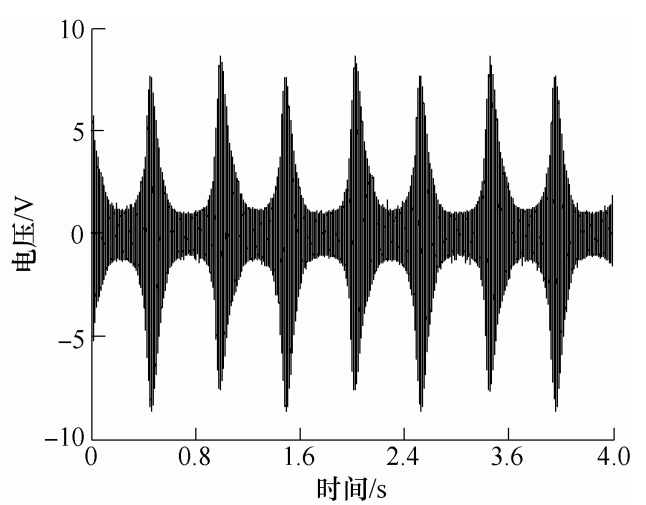

(a) 试件内磁通量密度

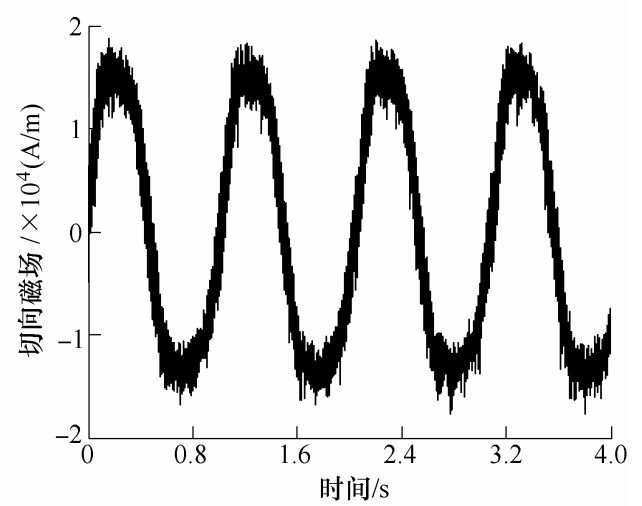

(b) 试件表面切向磁场

图 9 在线情况下磁混频传感器采集到的典型检测信号

显低于离线情况下的检测结果。根据疲劳损伤演化 的机理 ${ }^{[28-29]}$, 分析可能的原因为, 在疲劳试件制备 过程中, 虽然暂停了循环加载, 但金属材料内原子 的热扰动仍比较剧烈, 会影响检测信号的信噪比。 而离线试验是在疲劳试件静置 $48 \mathrm{~h}$ 后进行, 此时材 料内部原子的运动逐渐趋于平衡, 因此检测信号的 信噪比较高。

\section{3 试验结果及讨论}

对上述混频检测试验获得的检测信号进行分析 处理, 提取出混频非线性因子和局部磁滞损耗幂律 系数, 分析其与试件疲劳程度的关系。 


\section{1 离线检测}

对离线检测试验得到的磁通量密度信号进行傅 里叶变换, 得到图 10 所示的频谱图。可以看出, 对 于不同疲劳程度的试件, 对应检测信号的高频分量 $f_{1}=200 \mathrm{~Hz}$ 附近均存在多阶混频分量, 且难以直观观 察到低阶和频与差频分量幅值随试件疲劳程度的变 化规律。根据式(6)定义的磁混频非线性因子, 计算 不同疲劳试件对应的非线性因子, 得到图 11 所示磁 混频非线性因子随试件疲劳周期的变化关系。

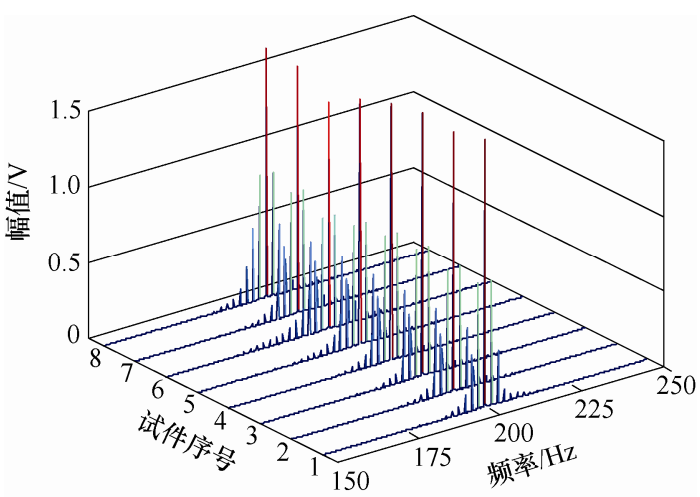

图 10 不同疲劳试件磁混频检测信号频谱图

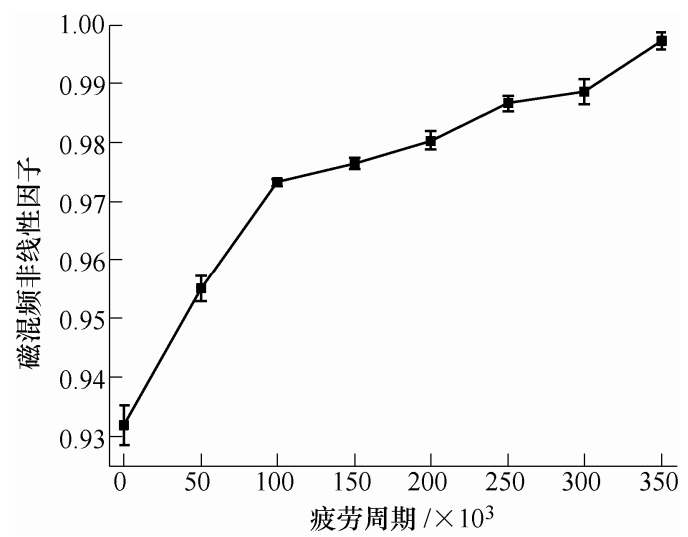

图 11 磁混频非线性因子随疲劳程度变化关系

从图 11 可以看出, 磁混频非线性因子随试件疲 劳周期数的增加呈单调递增规律。同时发现, 在疲 劳初期阶段(即疲劳周期小于 $10^{5}$ 时), 磁混频非线性 因子对疲劳周期变化更为敏感。

分别以磁混频传感器检测到的磁通量密度为 纵坐标、切向磁场为横坐标, 可以得到磁混频检测 的磁滞回线。图 12 给出了 8 个不同疲劳试件的磁滞 回线。

从图 12 可以看出, 磁混频检测得到的磁滞回线中 包含主磁滞回线与局部磁滞回线, 且主磁滞回线轮廓 平滑, 局部磁滞回线整齐。从直观上, 难以分辨出主 磁滞回线的形状和局部磁滞回线随试件疲劳程度的 变化规律。为此, 从混频检测得到磁滞回线中分别提 取主磁滞回线的三个特征参数(饱和磁化强度、剩磁强 度和矫顽力)与局部磁滞损耗幂律系数, 研究这些特征
参数随试件疲劳周期的变化关系, 结果如图 13 所示。

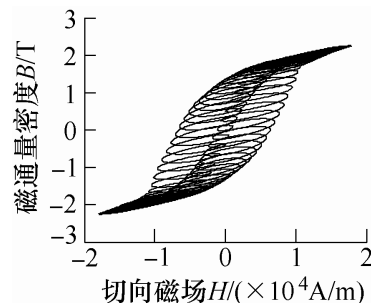

(a) 1 号试件

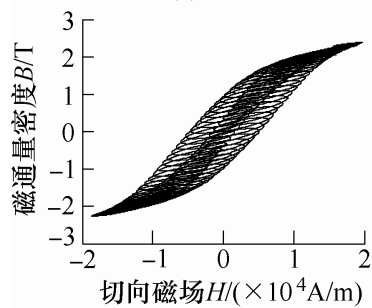

(c) 3 号试件

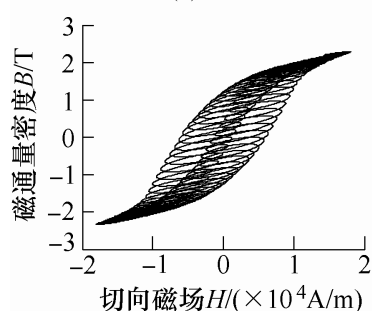

(e) 5 号试件

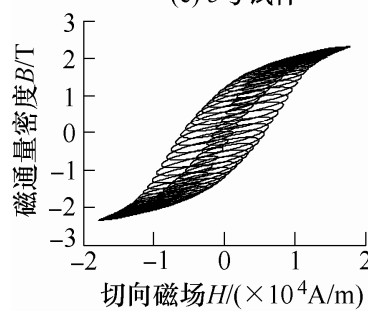

(g) 7 号试件

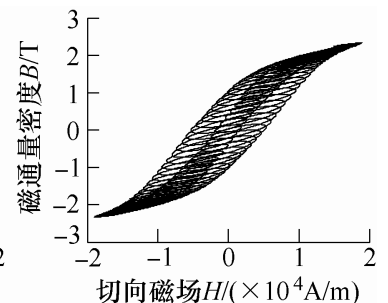

(b) 2 号试件

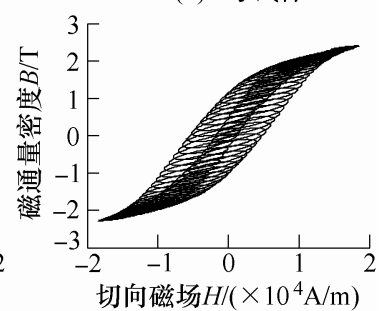

(d) 4 号试件

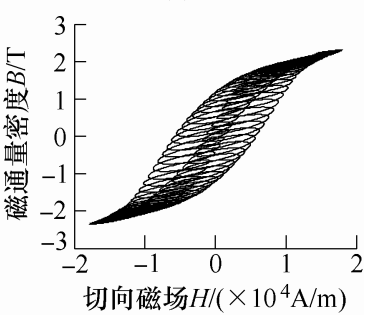

(f) 6 号试件

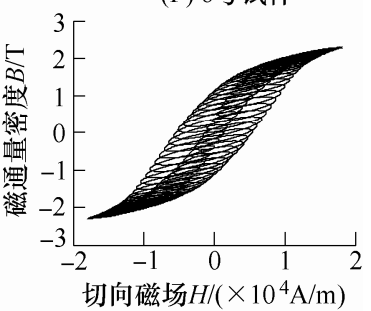

(h) 8 号试件
图 12 不同疲劳程度试件的磁混频检测磁滞回线

从图 13 可以看出, 这四个特征参数随试件 疲劳周数增加呈现不同的变化规律。其中，从主 磁滞回线中提取出的三个特征(饱和磁感应强度 $B_{s}$ 、剩磁强度 $B_{r}$ 和矫顽力 $H_{c}$, 如图 13a、13b 和 $13 \mathrm{c}$ 所示)随疲劳周期数的增加未呈现出明显的 规律，难以用于试件疲劳程度的表征。而局部 磁滞回线损耗幂律系数(图 13d) 随试件疲劳周数 的增加呈单调递减规律, 可以用于试件疲劳程度的 表征。

观察图 13d 和图 11 可以看出, 随试件疲劳程度 的增加, 本文提出的两种磁混频特征参数呈分段线 性变化趋势。具体说来，在疲劳加载周期低于 $1 \times$ $10^{5}$ 时, 两特征参数随疲劳周数增加变化较为急剧 (即磁混频非线性因子急剧增加, 局部磁滞损耗幂律 系数急剧减小); 当疲劳周数大于 $1 \times 10^{5}$ 时, 磁混 频非线性因子随疲劳周数的增加变得相对平缓; 当 疲劳周数在 $1 \times 10^{5}$ 到 $2 \times 10^{5}$ 范围时，局部磁滞损 耗幂律系数随疲劳周数增加变得较为平缓, 而当疲 


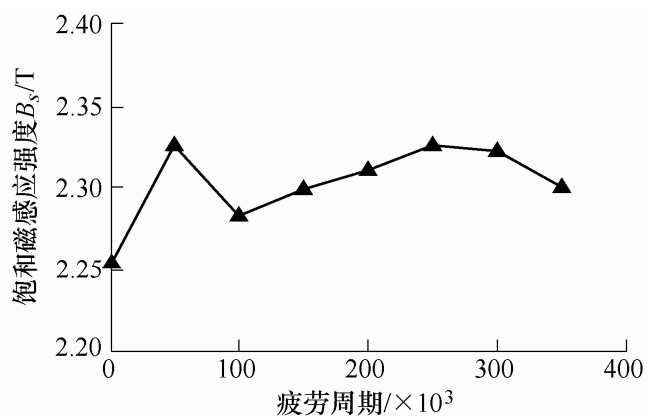

(a) 饱和磁感应强度

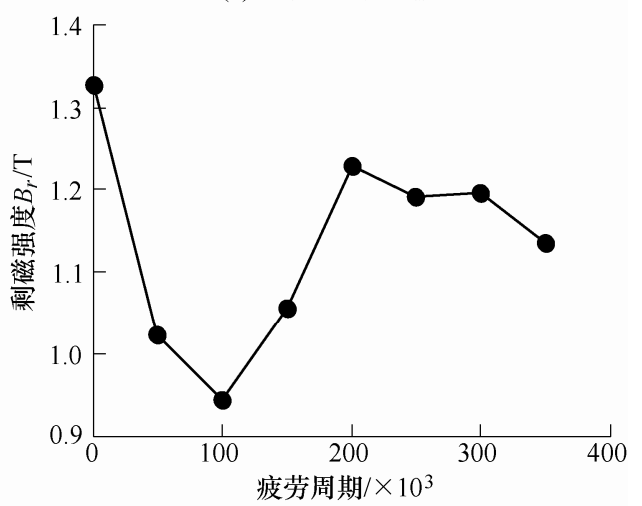

(b) 剩余磁感应强度

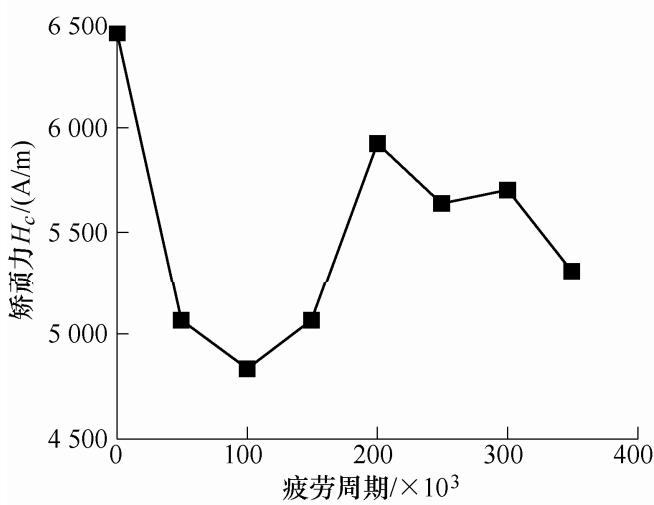

(c) 矫顽力

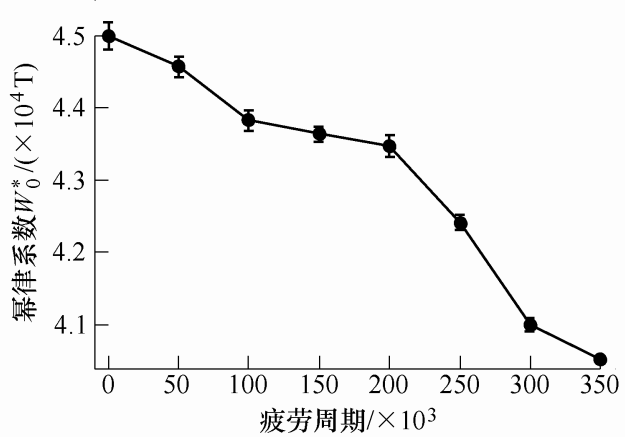

(d) 局部磁滞损耗幂律系数

图 13 磁滞回线特征参数随疲劳周期变化规律

劳周数大于 $1 \times 10^{5}$ 时, 局部磁滞损耗幂律系数随疲 劳周数增加又变得较为剧烈。两种特征参数与疲 劳周数增加呈分段线性变化规律, 可以通过材料 疲劳过程中其微观结构与性能变化给以解释。根 据金属材料的疲劳损伤机理可知, 在循环疲劳加载 作用下, 45 钢试件中部分晶粒的位错组态发生变 化, 产生不均匀滑移 ${ }^{[30]}$ 。在宏观上, 使试件呈现较
差的应变硬化能力和较弱的变形均匀分配能力, 即 会导致试件发生循环软化 ${ }^{[31]}$ 。通常, 在疲劳裂纹 形成前, 材料的循环软化会呈现多个阶段, 包括 初次软化, 随后减弱, 再二次软化的过程 ${ }^{[32]}$ 。 因此, 提出的两个特征参数随疲劳周数的分段线 性变化规律与试件疲劳过程的循环软化过程相 似，可用于材料疲劳损伤表征。

\section{2 在线检测}

按照相似的过程, 对在线检测数据进行处理, 图 14 给出了同一试件在 9 个不同疲劳阶段进行在 线检测得到的混频检测信号的频谱。与离线检测 结果图 9 相比, 在线检测信号的频谱较为杂乱, 低阶混频分量尚可分辨, 高阶混频分量已被掩 埋, 且频谱幅值较低。其原因如前所述, 在此不 再赘述。

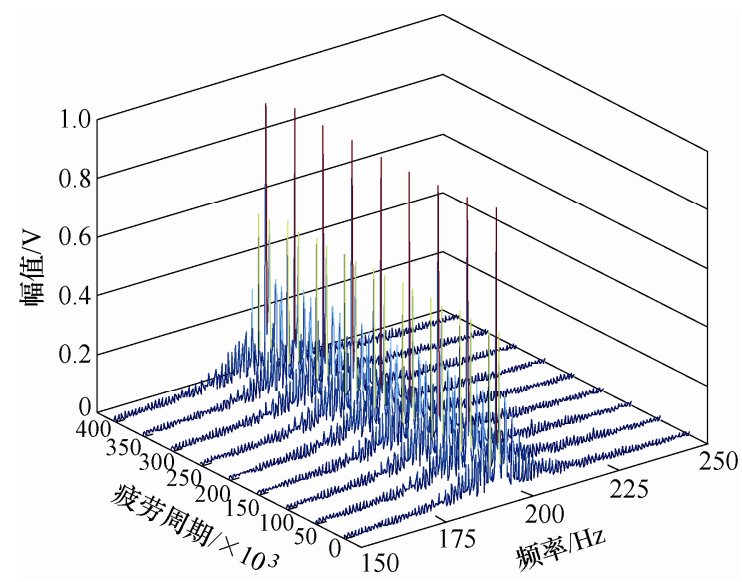

图 14 不同疲劳阶段磁混频检测信号的频谱图

图 15 给出了在线检测试验获得的磁混频非线 性因子随试件疲劳周期数的变化规律。分析可知, 磁混频非线性因子 $M$ 随试件疲劳周期的增加呈单 调增加规律, 这与离线结果相一致。但受信噪比影 响, 在线检测结果的分散性较大。

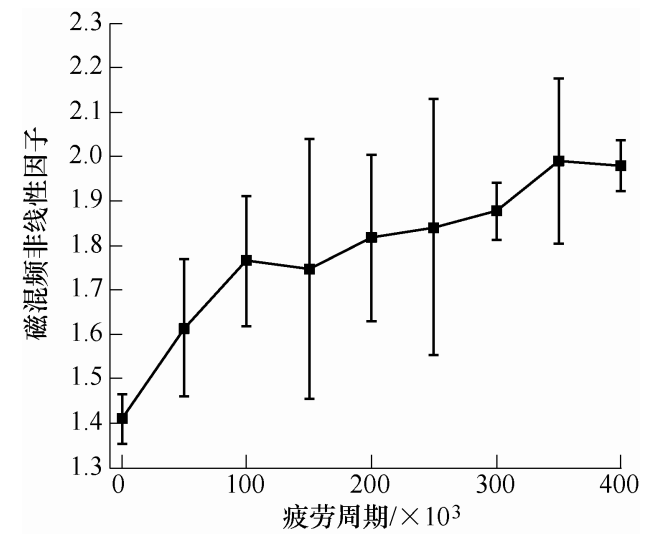

图 15 磁混频非线性因子随疲劳周期数的变化

类似地, 图 16 给出了磁混频在线检测的典型磁 
滞回线。与图 12 中离线检测的磁滞回线相比, 在线 检测所得磁滞回线, 其主磁滞回线轮廓不平滑, 局 部磁滞回线较为杂乱, 且在第一象限的饱和磁化处, 主磁滞回线轮廓未闭合。其原因亦是受限于检测信 号信噪比低的影响。

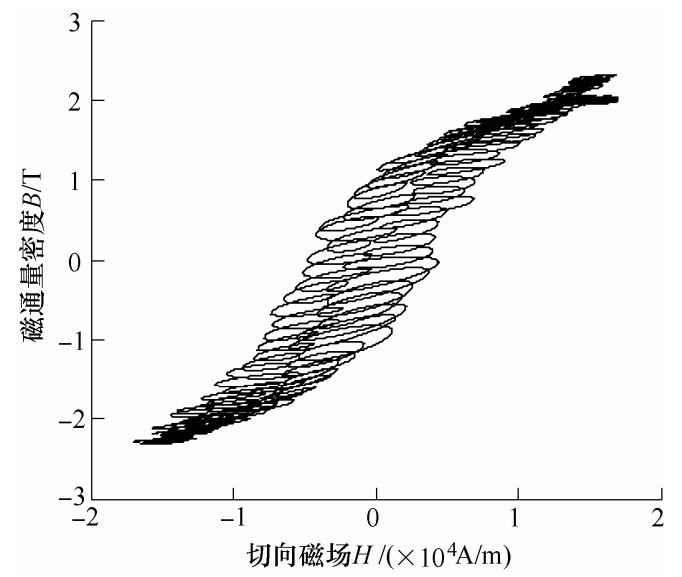

图 16 磁混频在线检测磁滞回线

图 17 进一步给出在线检测试验得到的局部磁 滞损耗幂律系数随试件疲劳周期数的变化关系。

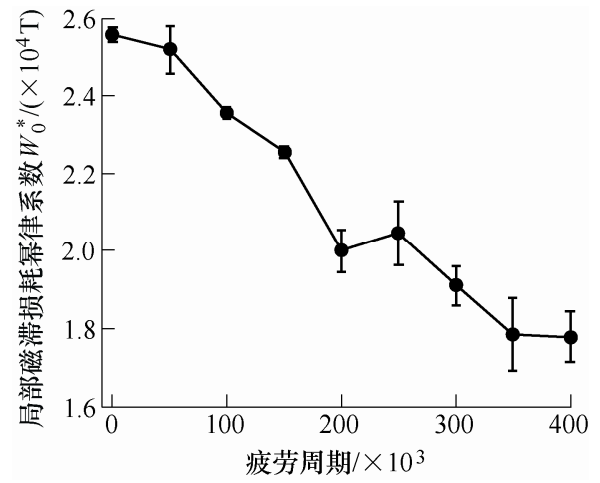

图 17 局部磁滞损耗幂律系数表征疲劳断裂过程

从图 17 中可以看出, 随疲劳程度的增加局部磁 滞损耗幂律系数呈近似单调递减变化。但与图 15 中的磁混频非线性因子相比, 该系数受磁混频检测 信噪比影响较小, 表现为结果的分散性较小。但与 图 13d 中离线检测的结果相比, 在线检测结果的分 散性仍略大。

综上所述, 提出的磁混频非线性因子和局部磁 滞损耗幂律系数两个特征参数均可用于试件疲劳程 度的表征。两个特征参数分别代表检测信号的频谱 特征和磁滞回线特征, 从两个方面反映了磁混频效 应随材料疲劳周期的变化情况。由于两个参数随疲 劳周期的变化趋势不同，使得两个参数敏感的疲劳 阶段不同。此外，两个参数的表征效果同时受检测 信号信噪比的影响, 但影响程度也不同。对表征结 果的鲁棒性而言, 局部磁滞损耗幂律系数更适用于 疲劳断裂过程的在线检测。

\section{4 结论}

针对铁磁性构件早期疲劳损伤检测问题, 本文 发展了一种磁混频无损检测技术, 得出以下结论。

(1) 提出的磁混频非线性因子与磁混频局部磁 滞损耗幂律系数均可用于铁磁性构件早期疲劳损伤 的表征。

(2) 两种磁混频特征参数受噪声影响程度不 同。局部磁滞损耗幂律系数受信噪比影响更小, 更 适合于复杂环境下试件疲劳评价。

(3) 磁混频非线性因子与局部磁滞损耗幂律系 数随试件疲劳加载周期的变化, 呈现与材料疲劳软 化相似的分段变化过程。

\section{参 考 文 献}

[1] STINVILLE J C, MARTIN E, KARADGE M, et al. Fatigue deformation in a polycrystalline nickel base superalloy at intermediate and high temperature : Competing failure modes[J]. Acta Materialia, 2018, 152(15): 16-33.

[2] 严园, 刍兰林, 周兴林. 钢桥疲劳裂缝的红外热成像无 损检测 $[J]$. 应用科学学报，2016，34(1): 106-114.

YAN Yuan, ZOU Lanlin, ZHOU Xinglin. Nondestructive inspection of steel bridge based on infrared thermal imaging[J]. Journal of Applied Sciences, 2016，34(1): 106-114.

[3] WANG Y, GAO B, WOO W L, et al. Thermal pattern contrast diagnostic of micro cracks with induction thermography for aircraft braking components[J]. IEEE Transactions on Industrial Informatics, 2018, 14(12): 5563-5574.

[4] CHAI M, ZHANG Z, DUAN Q, et al. Assessment of fatigue crack growth in $316 \mathrm{LN}$ stainless steel based on acoustic emission entropy[J]. International Journal of Fatigue, 2018, 109(4): 145-156.

[5] JIAO J, CHANG Y, WU C, et al. Detection of heterogeneous deposits on the surface of metal structures using nonlinear acoustic resonance technology[J]. Surface Toopgraphy: Metrology and Properties, 2017, 5(4): 1-11.

[6] WANG K, LIU M, SU Z, et al. Analytical insight into "Breathing" crack-induced acoustic nonlinearity with an application to quantitative evaluation of contact cracks[J]. Ultrasonics, 2018, 88(8): 157-167.

[7] NI C, HUA L, WANG X. Crack propagation analysis and fatigue life prediction for structural alloy steel based on metal magnetic memory testing $[\mathrm{J}]$. Journal of Magnetism and Magnetic Materials, 2018, 462(1): 144-152. 
[8] JILES D C, ATHERTON D L. Theory of magnetic hysteresis[J]. Journal of Magnetism \& Magnetic Materials, 1986, 61(2): 48-60.

[9] JILES D C, MELIKHOV Y. Modelling of nonlinear behaviour and hysteresis in magnetic materials[M]// Handbook of Magnetism and Advanced Magnetic Materials. John Wiley \& Sons, Ltd, 2007: 71-81.

[10] SIMA W, ZOU M, YANG M, et al. Modeling of grain-oriented Si-steel and amorphous alloy iron core under ferroresonance using Jiles-Atherton hysteresis method[J]. AIP Advances, 2018, 8(5): 1-6.

[11] BAGHEL A P S, SHEKHAWAT S K, KULKARNI S V, et al. Modeling of dynamic hysteresis for grain-oriented laminations using a viscosity-based modified dynamic Jiles-Atherton model[J]. Physica B Physics of Condensed Matter, 2014, 448(1): 349-353.

[12] SAMIMI A A, KRAUSE T W, CLAPHAM L. Stress response of magnetic barkhausen noise in submarine hull steel: a comparative study[J]. Journal of Nondestructive Evaluation, 2016, 35(2): 1-6.

[13] MATSUMOTO T, UCHIMOTO T, TAKAGI T, et al. Evaluation of chill structure in ductile cast iron by incremental permeability method[J]. Internation Journal of Applied Electromagnetics and Mechanics, 2016, 52(6): 1599-1605.

[14] CHEN H E, XIE S, ZHOU H, et al. Numerical simulation of magnetic incremental permeability for ferromagnetic material[J]. International Journal of Applied Electromagnetics \& Mechanics, 2014, 45(1): 379-386.

[15] 陈洪恩, 陈振茂, 李勇, 等. 基于磁噪声和增量磁导率 的塑性变形定量无损评价 [J]. 无损检测, 2012, 34(10): 12-15.

CHEN Hongen, CHEN Zhenmao, LI Yong, et al. Quantitative NDE of plastic deformation based on barkhausen noise and incremental permeability[J]. Nondestructive Testing, 2012, 34(10): 12-15.

[16] ZIRKA S E, MOROZ Y I, MARKETOS P, et al. Viscosity-based magnetodynamic model of soft magnetic materials[J]. IEEE Transactions on Magnetics, 2006, 42(9): 2121-2132.

[17] KOBAYASHI S , ISHIBASHI Y , BABA R. Re-examination of the Steinmetz law for unsymmetrical magnetic hysteresis loops[J]. Journal of Magnetism \& Magnetic Materials, 2013, 330(5): 49-54.

[18] TAKAHASHI S, KOBAYASHI S, KIKUCHI H, et al. Relationship between mechanical and magnetic properties in cold rolled low carbon steel[J]. Journal of Applied Physics, 2006, 100(11): 979-985.

[19] YU C, JINGPIN J, XIUCHENG L, et al. Measurement of the hardness of medium carbon steel using the magnetic mixing-frequency technique[J]. IEEE Transactions on Magnetics, 2018, 54(12): 1-7.

[20] FICKO B W, GIACOMETTI P, DIAMOND S G. Nonlinear susceptibility magnitude imaging of magnetic nanoparticles[J]. Journal of Magnetism \& Magnetic Materials, 2015, 378(15): 267-277.

[21] XU H, PEI Y, FANG D, et al. Nonlinear harmonic distortion effect in magnetoelectric laminate composites[J]. Applied Physics Letters, 2014, 105(1): 417-421.

[22] RABEHI A, GARLAN B, ACHTSNICHT S, et al. Magnetic detection structure for lab-on-Chip applications based on the frequency mixing technique[J]. Sensors, 2018, 18(6): 1747-1760.

[23] BURDIN D A, CHASHIN D V, EKONOMOV N A, et al. Resonance mixing of alternating current magnetic fields in a multiferroic composite[J]. Journal of Applied Physics, 2013, 113(3): 101-106.

[24] BURDIN D A, CHASHIN D V, EKONOMOV N A, et al. Nonlinear magneto-electric effects in ferromagneticpiezoelectric composites[J]. Journal of Magnetism \& Magnetic Materials, 2014, 358-359(5): 98-104.

[25] KRAUSE H J, WOLTERS N, ZHANG Y, et al. Magnetic particle detection by frequency mixing for immunoassay applications[J]. Journal of Magnetism \& Magnetic Materials, 2007, 311(1): 436-444.

[26] TELIBAN I, THEDE C, CHEMNITZ S, et al. Magnetic moment investigation by frequency mixing techniques.[J]. Review of Scientific Instruments, 2009, 80(11): 631-635.

[27] TU L, FENG Y, KLEIN T, et al. Measurement of Brownian Relaxation of Magnetic Nanoparticle by a Multi-Tone Mixing-Frequency Method[J]. IEEE Transactions on Magnetics， 2012，48(11): 3513-3516.

[28] 许金泉, 郭风明. 疲劳损伤演化的机理及损伤演化律 [J]. 机械工程学报, 2010, 46(2): 40-46.

XU Jinquan, GUO Fengming. Mechanism of fatigue damage evolution and the evolution law[J]. Journal of Mechanical Engineering, 2010， 46(2): 40-46.

[29] YANG Y J, XING L Y, DUAN H Q, et al. Fatigue damage evolution of coal under cyclic loading[J]. Arabian Journal of Geosciences, 2018， 18(11): 560-572.

[30] 杨浩泉, 周平. 高周疲劳过程中 45 钢表面显微硬度变 化规律的研究[J]. 理化检验-物理分册, 2006, 42(7): 328-331.

YANG Haoquan, ZHOU Ping. Study on the characteristic of the surface microhardness for 45 steel during high cycle fatigue[J]. Physical Testing and Chemical Analysis Part A: Physical Testing, 2006, 42(7): 328-331.

[31] LODE V. Magnetic hysteretic characterization of 
ferromagnetic materials with objectives towards non-destructive evaluation of material degradation[M]. Gent: Universiteit Gent, 2009.

[32] 王光中, 柯伟. 金属疲劳的微观过程 [J]. 材料科学与工 程, 1984(3): 28-38.

WANG Guangzhong, KE Wei. The microscopic process of metal fatigue $[\mathrm{J}]$. Materials Science and Engineering,
1984(3): 28-38.

作者简介: 焦敬品(通信作者), 女, 1973 年出生, 博士，教授，博士研 究生导师。主要研究方向为现代测控技术与方法、无损检测新技术、现 代信号分析与处理技术、新型传感器技术。

E-mail: jiaojp@bjut.edu.cn

常予, 女, 1991 年出生, 博士研究生。主要研究方向为电磁混频无损检 测技术与方法。

E-mail: changyu8023@163.com 